Submitted to ApJ

Preprint typeset using LTEX style emulateapj v. 5/2/11

\title{
INTRA-BINARY SHOCK HEATING OF BLACK WIDOW COMPANIONS
}

\author{
Roger W. ROMANi \& NiCOLAS SANCHEZ ${ }^{1}$ \\ Submitted to ApJ
}

\begin{abstract}
The low mass companions of evaporating binary pulsars (black widows and their ilk) are strongly heated on the side facing the pulsar. However in high-quality photometric and spectroscopic data the heating pattern does not match that expected for direct pulsar illumination. Here we explore heating mediated by an intrabinary shock (IBS). We develop a simple analytic model and implement it in the popular 'ICARUS' light curve code. The model is parameterized by the wind momentum ratio $\beta$ and velocity $v_{\mathrm{Rel}} v_{\mathrm{orb}}$ and assumes that the reprocessed pulsar wind emits prompt particles or radiation to heat the companion surface. We illustrate an interesting range of light curve asymmetries controlled by these parameters. The code also computes the IBS synchrotron emission pattern, and thus can model black widow X-ray light curves. As a test we apply the results to the high quality asymmetric optical light curves of PSR J2215+5135; the resulting fit gives a substantial improvement upon direct heating models and produces an X-ray light curve consistent with that seen. The IBS model parameters imply that, at the present loss rate, the companion evaporation has a characteristic timescale $\tau_{\text {evap }} \approx 150 \mathrm{My}$. Still, the model is not fully satisfactory, indicating additional unmodeled physical effects.
\end{abstract}

Subject headings: gamma rays: stars — pulsars: general

\section{INTRODUCTION}

Since discovery of the original 'black widow' pulsar PSR J1959+2048, it has been realized that optical study of the pulsar companion provides an important path to understanding the dynamics of these exotic systems, including the pulsar heating mechanism, the companion wind and the component masses (Diorgovski \& Evans 1988; Aldcroft, Romani \& Cordes 1992; Callanan, van Paradijs \& Rengelink 1995). Such studies became even more interesting when van Kerkwijk, Breton, \& Kulkarni (2011) found evidence that this pulsar might be especially massive, so that precision measurements of black widow component masses could have important implication for our understanding of the binary evolution and of the dense matter equation of state.

The quest seems straight-forward. Radio or gamma-ray pulsar timing provides a precise orbital ephemeris and a companion mass function, via the orbital period $P_{B}$ and projected semi-major axis of the pulsar orbit $x=a_{p} \sin i$ :

$$
f\left(M_{P}, q, i\right)=\frac{4 \pi^{2} x^{3}}{G P_{B}^{2}}=M_{P} \frac{(\sin i)^{3}}{q(q+1)^{2}}
$$

with $M_{P}$ and $M_{C}$ the pulsar and companion masses and $q=$ $M_{P} / M_{C}$ the mass ratio. In clean double-degenerate systems, relativistic effects in precision pulsar timing allow solution for $q$ and $i$. For the companion-evaporating pulsars (black widows, with $M_{C} \approx 0.01-0.03 M_{\odot}$, redbacks with $M_{C} \approx$ $0.1-0.3 M_{\odot}$, and their ilk) the dissipation and propagation effects of the companion and wind preclude such precision timing. However, optical studies of the companion can, in principle, measure the spectroscopic radial velocity amplitude $K_{C}$ (giving $q=K_{C} P_{B} / 2 \pi x$ ) and, by measuring the optical modulation due to varying view of the heated side, the orbital inclination $i$.

The challenge is that the radial velocity observed is weighted toward the center of light on the heated side

\footnotetext{
${ }^{1}$ Department of Physics, Stanford University, Stanford, CA 94305 4060, USA; rwr@astro.stanford.edu
}

so that $K_{C}=K_{o b s} K_{c o r r}$ is larger that the observed radial velocity amplitude by $K_{\text {cor }} \approx 1.03-1.08 \times$ (Romani, Filippenko \& Cenko 2015), depending on $i$ and the heating pattern. One commonly assumes that the pulsar spindown power heats the facing side of the companion directly, raising the characteristic temperature from the unheated ('Night' side) $T_{N}$ to

$$
T_{D}^{4}=\eta \dot{E} / 4 \pi a^{2} \sigma+T_{N}^{4}
$$

with $a=x(1+q) / \sin i$ the orbital separation, $\dot{E}=$ $I \Omega \dot{\Omega}$ the pulsar spindown power for moment of inertia $I$ and $\eta$ a heating efficiency. This model has been implemented in several light curve modeling codes eg. the ELC code (Orosz \& Hauschildt 2000) and its descendant ICARUS (Breton et al. 2013). Direct isotropic 'photon' heating is assumed, which is indeed a good approximation for many X-ray binaries. Fitting black widow light curves and spectra with such codes has led to surprisingly large estimates of $M_{N}: 2.4 \pm 0.12 M_{\odot}$ for PSR J1959+2048 (van Kerkwiik, Breton, \& Kulkarni 2011) and $\approx 2.7 M_{\odot}$ for PSR J1311-3430 (Romani, Filippenko \& Cenko 2015).

However, with the discovery of a large population of BW and RB in the direction of Fermi sources, several nearby, bright systems have been found, enabling high precision optical light curves and spectroscopy. Direct heating models, which adequately described some early low precision observations, often do not provide a statistically acceptable description of the high precision data. In particular, many light curves are substantially asymmetric (Stappers et al. 2001; Schroeder \& Halpern 2014), the color and spectral variations across the face do not match direct heating patterns (Romani, Filippenko \& Cenko 2015) and the inferred heating power in several cases show large $\eta \geq 1$. This implies that the pulsar power does not heat the companion via direct illumination but that pulsar particles or high energy radiation are deflected before reaching the companion. In one natural scenario the pulsar and companion winds set up an intra-binary shock (IBS); the heating power arises in this structure. In fact the X-ray light curves of many BW and RB show modulation 


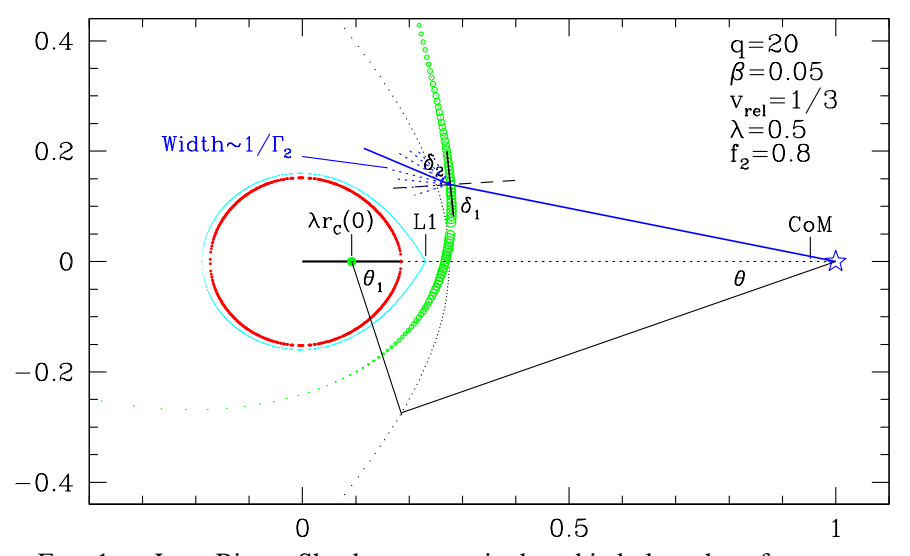

FIG. 1. - Intra-Binary Shock geometry in the orbital plane, here for a mass ratio $q=20$ (RB-like), a wind momentum ratio $\beta=0.05$ and a relative velocity $v_{\text {Rel }}=1 / 3$. The wind origin is placed halfway toward the companion 'nose' facing the $L_{1}$ point. The wind angles $\theta_{1}, \theta_{2}$ are shown for the $v_{\mathrm{Rel}}=\infty$ case, without sweep-back. The local intensity of the swept shock surface is indicated by point size and the shock inclination angles and post-shock beaming are shown for one ray.

indicating such IBS (Roberts et al. 2014). A second plausible picture invokes a companion magnetic field intercepting the pulsar wind and channeling spindown power to the surface. Since companion magnetic structures are at present poorly constrained, we focus here on a pseudo-analytic model for IBS-mediated illumination, allowing robust fits for the principal physical wind parameters in data fitting codes. We have developed an ICARUS module employing this model. The results can mimic a range of observed $\mathrm{BW} / \mathrm{RB}$ behavior and result in dramatically improved light curve fits. However, we show that some aspects are not adequately modeled and close by briefly describing additional physical ingredients, such as companion fields, likely needed in the fits.

\section{INTRA-BINARY SHOCK MODEL}

Although existing codes such as ICARUS assume isotropic pulsar irradiation, the relativistic wind is likely equatorially concentrated as $\propto \sin ^{2} \theta$, with $\theta$ the polar angle

$$
f_{P}(r, \theta)=\dot{E}(\theta) /\left(4 \pi r^{2} c\right)=3 I \Omega \dot{\Omega} \sin ^{2} \theta /\left(4 \pi r^{2} c\right) .
$$

In fact Tchekhovskoy, Spitkovsky \& Li (2013) find that, for large pulsar inclination angle $\alpha$, numerical simulations show a wind power distributed as approximately $\sin ^{4} \theta$. We will assume a quadratic form here, and compare with the $\theta^{0}$ and $\theta^{4}$ cases. This wind shocks against a baryonic companion wind of speed $v_{W}$ and mass loss rate $\dot{M}_{W}$, which gives rise to a momentum flux

$$
f_{C}(r)=\dot{M}_{W} v_{W} /\left(4 \pi r^{2}\right) .
$$

We will assume that this wind is isotropic. Thus the wind shock geometry is controlled by two principal parameters, the wind momentum flux ratio

$$
\beta=\dot{M}_{W} v_{W} c / \dot{E}
$$

and the ratio between the massive wind speed and the orbital velocity $v_{\text {Rel }}=v_{W} / v_{\text {orb }}$. The companion wind is driven (in a poorly understood way) by pulsar irradiation. Further, in the Roche geometry the escape potential is lowest at the L1 point. These effects suggest a companion wind centered inward of its center of mass. We parametrized this shift with a secondary parameter $\lambda$, with $\lambda=0$ for a wind centered on the star and $\lambda=1$ centered on the star surface at the sub-pulsar point closest to L1 $\left(r_{C}(0)\right.$ from the companion center). This parameter does not have a strong effect unless the companion wind is quite weak ( $\operatorname{small} \beta)$.

We compute the contact discontinuity surface (implicitly assuming a thin shell IBS, with rapid cooling). The wind origins are at the pulsar and a distance $d=a-\lambda r_{C}(0)$ toward the companion along the binary axis. This locates the IBS as a surface of revolution about the binary axis with

$$
r(\theta)=d \sin \theta_{1} / \sin \left(\theta+\theta_{1}\right)
$$

(Cantó, Raga \& Wilkin 1996), where $\theta$ is the angle between the line of centers and the ray from the pulsar and

$$
\theta_{1}=\left[\frac{15}{2}\left(\left[1+\frac{4}{5} \beta(1-\theta \cot \theta)\right]^{1 / 2}-1\right)\right]^{1 / 2}
$$

is the equivalent angle from the companion wind center (Figure 1). This describes the intersection of two stationary winds. In our case the motion of the companion causes the shock symmetry axis to trace an Archimedean spiral (Parkin \& Pittard 2008), lagged in true anomaly behind the center of mass position by an orbital phase angle $\delta \phi_{B}(r) \approx r /\left(2 \pi d v_{\mathrm{Rel}}\right)$. The resulting geometry compares well with shock structures seen in numerical simulations (e.g. Bosch-Ramon, Barkov \& Perucho 2015) and the two parameter family captures the range of wind ratios and orbital distortion, while remaining quickly calculable.

The pulsar wind has a transverse embedded field, whose magnetization parameter $\sigma=B^{2} /\left(4 \pi \gamma \rho c^{2}\right)$ is poorly known. Without reconnection, the transverse toroidal field should have a value $B(r) \approx 4 B_{P}(2 \pi / c P)^{2} r_{N S}^{3} / r$ in the post shock region. For a typical MSP surface dipole $B=10^{8} B_{8} \mathrm{G}$ spin period $P=3 P_{3} \mathrm{~ms}$ and orbital separation $a \sim 10^{11} \mathrm{~cm}$, this is $B_{I B S} \approx 20 B_{8} a_{11}^{-1} P_{3}^{-2} \mathrm{G}$. Away from the sub-pulsar point the shock is oblique with an angle to the shock decreasing from $\delta_{1}$ to a post-shock $\delta_{2}=\tan ^{-1} \chi(\sigma) \delta_{1}$ (Figure 1) with

$$
\chi(\sigma)=\left[1+2 \sigma+\left(16 \sigma^{2}+16 \sigma+1\right)^{1 / 2}\right] /(6+6 \sigma)
$$

and post-shock bulk $\Gamma_{2}=\left(1-\chi^{2}\right)^{-1 / 2} / \sin \delta_{1}$ (Komissarov \& Lyutkov 2011). These relations hold for ultra-relativistic transverse field flow, even when the field is not in the shock plane (Y. Yuan, private communication). Given our poor knowledge of the wind properties we assume $\sigma=1$ in the following. We have explored the magnetization dependence; in principle a well understood shock geometry allows a probe of this important parameter, but we will not discuss that dependence here.

In the spirit of our thin shock approximation, we assume prompt radiation from the shocked energetic pulsar wind. We take this to imply radiation in a Gaussian beam of width $\sigma_{r}=1 / \Gamma_{2}$ centered around the immediate post-shock flow vector $\pi / 2-\delta_{2}$ to the local shock normal. If we assume that the full power of the pulsar wind impacting on the IBS $f_{p}(r, \theta)$ is promptly re-radiated from $e^{ \pm}$at large pitch angle, it emits $\approx f_{p}(r, \theta) \cos \left(\pi / 2-\delta_{1}\right) / \Gamma_{2}$ of the wind energy per unit area. With the $B_{I B S}$ above, the bright IBS X-rays come from particles with $\gamma \approx 5 \times 10^{4}\left(E_{\mathrm{keV}} / B_{I B S}\right)^{1 / 2}$ and a cooling time $\tau \approx 30 E_{k e V}^{-1 / 2} B_{I B S}^{-3 / 2}$ s. With $B_{8} \sim 2-3$ this is comparable to the flow time near the stagnation point at the IBS apex where $t_{\text {flow }}>a /(c / 3) \sim 10$ s. Note that the shock is strong at the shock apex, so that $\Gamma_{2}$ is small and the radiation is widely beamed. In contrast, as the shock becomes 
tangential down stream (or as swept back by orbital motion) $\Gamma_{2}$ grows. Thus both the solid angle and shock weakening decrease the prompt-emission surface brightness, although this decreased emission is increasingly beamed closer to the shock limb.

Some post-shock radiation will not be prompt. Indeed most may be slow e.g. if strong reconnection drives $\sigma \ll 1$, reducing $B_{I B S}$, or if the immediate post-shock pitch angle is small. In this case the radiation persists as the shocked wind flows a distance $\sim a$. Then we may assume that the emission is directed approximately tangent to the contact discontinuity, and that the shocked pulsar wind accelerates as it flows away from the shock apex. Examining numerical simulations of Bogovalov et al. (2012) we can approximate the resulting bulk $\Gamma_{\|}$as

$$
\Gamma_{\|} \approx 1.2\left(1+d r / r_{0}\right)
$$

where $r_{0}$ is the standoff distance (along the line of centers) of the weaker wind and for a given position on the IBS $d r$ is the increase in radial distance from the stronger wind center to that along the line of centers (i.e. $d r=0$ at the nose). The numerical simulations do not give a clear prescription for the emissivity; the particle density drops rapidly behind the apex, but the magnetic field appears to initially grow in the post shock flow before downstream dilution. For simplicity and to compare with the prompt emission scheme, we assign surface brightness as above, scaled with the diminishing pulsar wind flux per unit IBS area; this mimics the downstream fading expected from the simulations.

These expressions for the shock emissivity and its reradiation heating of the companion surface have been implemented in the ICARUS code. This includes options to compute the optical companion light curve and the synchrotron IBS-dominated light curves for any desired inclination. The code also minimizes residuals with respect to optical data to determine model parameters and errors. While assembling these routines, we noted that the standard ICARUS distribution was missing a $\cos \chi$ (with $\chi$ the angle to the local surface normal) in the companion heating computation. There was also an error in the treatment of limb darkening. These have now been amended, but parameters fit with ICARUS before 2016 will likely need updating. The IBS modules and instructions for their inclusion will be posted on Github.

\section{GEOMETRY DEPENDENCE FOR THE IBS AND COMPANION} LIGHT CURVES

The basic IBS structure is set by $\beta$ and $v_{\text {Rel }}$. Small $\beta$ lie close to the companion surface, $\beta=1$ produces a flat, mid-orbit shock, for large $\beta$ the shock lies close to the pulsar. Large $v_{\text {Rel }}$ produces a nearly symmetric IBS, while for $v_{W}<v_{\text {orb }}$ the sweep-back is appreciable. In all cases, the most energy is intercepted near the IBS 'nose' and re-radiated to the companion surface.

Figure 2 shows the basic $\beta$ dependence for a relatively fast companion wind with $v_{\mathrm{Rel}}=3$, with the middle and bottom rows showing the skymap and three light curve cuts, respectively, for the direct (IBS synchrotron) emission of the 'slowcooling' tangential component. For small $\beta$ there is a substantial effect from eclipse by the companion (Bogdanov et al. 2011). However, for most models, the dominant effect is from Doppler beaming at the limb of the pulsar limb shock. The result is a double-peaked X-ray (synchrotron) light curve centered on optical minimum (MSP radio eclipse). For large $\beta$ (weak PSR, strong companion wind) Doppler beaming con- trols the light curve, which is now centered opposite the MSP radio eclipse. Note that there is substantial asymmetry even for the the relatively fast companion wind shown here; for small $v_{R e l}$ or small inclination $i$ often only a single peak appears.

In figure 3 we show two example X-ray light curves. The first is for the original black widow PSR J1959+2048 (Huang et al. 2012), which has a highly energetic pulsar and weak companion wind (small $\beta$ ). The second, J2129-0429 (Roberts et al. 2015) is a long period period, lower $\dot{E}$ redback with a relatively massive secondary $\sim 0.4 M_{\odot}$ undergoing quasi-Roche lobe overflow (qRLOF, Bellem et al. 2016). As such it plausibly has a rather high $\dot{M}_{W}$ and thus large $\beta$. This is similar to the situation seen for high-mass $\gamma$-ray binaries such as LS 5039.

For $\beta<1$, the prompt post-shock IBS emission illuminates and heats the companion. In figure 4 we show the shock geometry (now including varying $v_{R e l}$ ) and the optical light curves of the heated companion. The resulting heating pattern differences are not visually striking, but they introduce substantial light curve asymmetry, especially for small $\beta$ and $v_{\text {Rel }}$ The examples shown here have $\lambda=0.7$. This parameter has modest effect on the light curve shape unless $\beta$ is very small. The orbital sweep back for very small $v_{\text {Rel }}$ can wrap the IBS around the companion; we assume that the portion of the IBS beyond the tangent point (companion backside) does not intercept pulsar flux or radiate. As expected, $v_{R e l}$ dominates the light curve asymmetry and the sensitivity to the heating pattern is strongest for blue colors.

\section{APPLICATION TO PSR J2215+5135}

PSR J2215+5135 is a redback (RB) system, a $P=2.6 \mathrm{~ms}$ $\dot{E}=7.4 I_{45} \times 10^{34} \mathrm{erg} \mathrm{s}^{-1}$ (with the neutron star moment of inertia $I_{45} 10^{45} \mathrm{~g} \mathrm{~cm}^{2}$ ) millisecond pulsar in a $P_{b}=$ $4.14 \mathrm{hr}$ orbit with $\mathrm{a} \sim 0.23 M_{\odot}$ companion. Schroeder \& Halpern (2014, hereafter SH14) obtained high-quality BVR light curves of the companion over many orbits, finding that the source varies from $V \approx 18.7$ to $20.2 \mathrm{mag}$, showing strong heating. Their fit with the ELC code and a photometry table generated from the PHOENIX model atmospheres (Husser et al. 2013) suggested small inclination $i$ and had a number of peculiarities, including poor agreement with the observed colors and a highly significant phase shift of optical maximum by $\Delta \phi \approx-0.01$ with respect to the radio-pulse ephemeris. Romani et al. (2015) obtained Keck LRIS spectra throughout the orbit and were thus able to greatly improve the model fits, finding a much larger inclination $i \approx 90^{\circ}$ (and hence much smaller component masses). Gentile et al. (2014) observed the system in the X-rays with $\mathrm{CXO}$, finding an X-ray minimum near orbital phase $\phi \approx 0$ (pulsar superior conjunction, optical minimum, radio eclipse), which they interpret as due to variable obscuration of emission from an intrabinary shock around the companion. Thus this system is a a good example to test our IBS model and indirect re-heating code.

We use here $103 \mathrm{~B}, 55 \mathrm{~V}$, and $113 R$ magnitudes from $\mathrm{SH} 14$. The radio pulsar timing gives us $x=a_{1} \sin i=$ $0.468141433 \mathrm{lt}-\mathrm{s}$ and accurate orbital ephemeris to phase the photometry points. The basic ICARUS model parameters are the underlying temperature of the star (actually $T_{N}$ of the unheated "night" face), a heating flux denoted $L_{H}$, the orbital inclination $i$ and the mass ratio $q$. Here

$$
L_{H}=\left(T_{D}^{4}-T_{N}^{4}\right) 4 \pi\left[x_{1}(1+q)\right]^{2} \sigma / \sin ^{2} i
$$




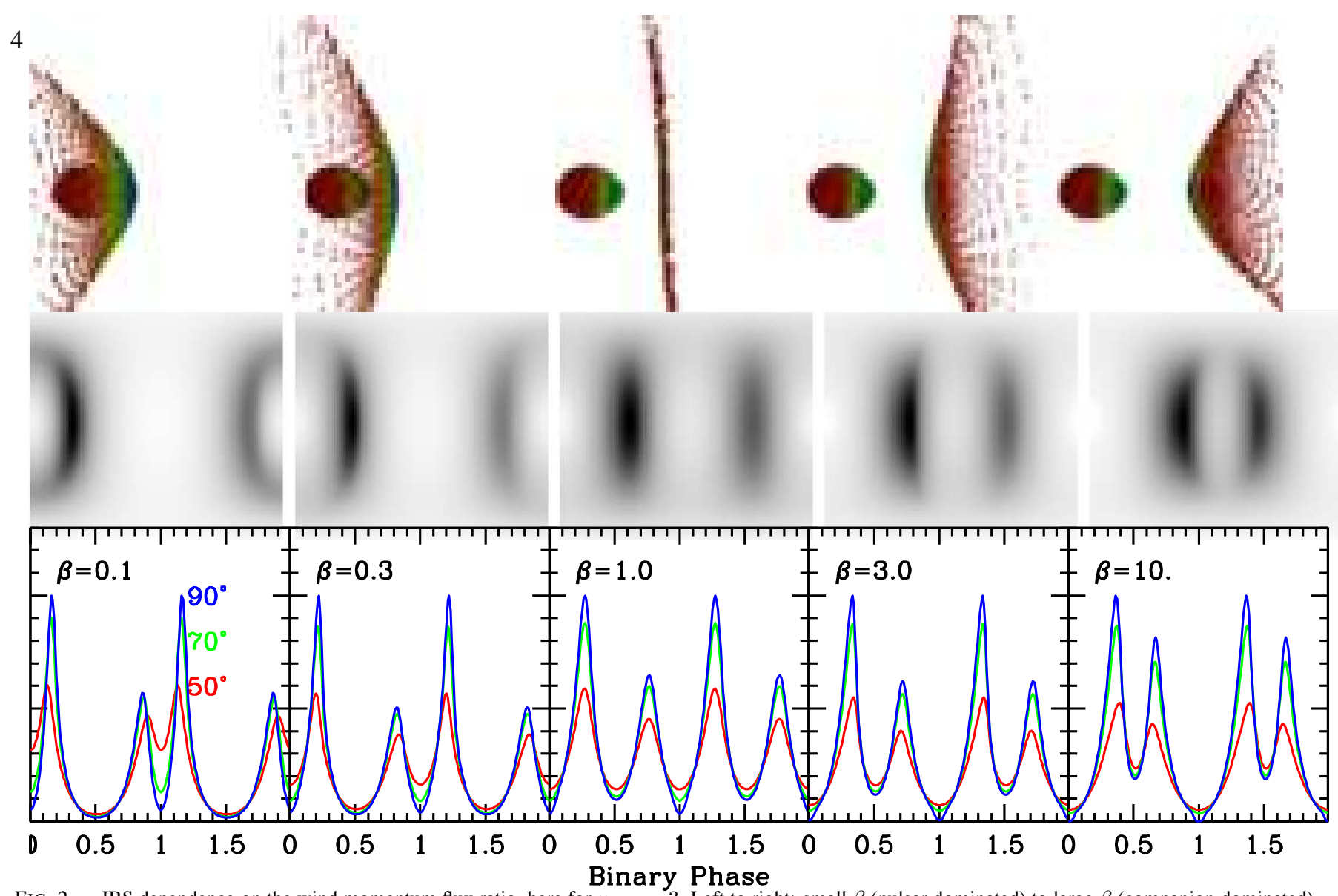

FIG. 2.- IBS dependence on the wind momentum flux ratio, here for $v_{\text {Rel }}=3$. Left to right: small $\beta$ (pulsar dominated) to large $\beta$ (companion dominated). Top: images of the heated companion and the IBS shock contact discontinuity, viewed from the orbital plane at $\phi_{B}=\pi / 2$. Middle: IBS Synchrotron emission pattern on the sky, with each panels covering $\phi_{B}=0$ (pulsar inferior conjunction) to $\phi_{B}=2 \pi$ and viewing angle $i=0$ to $\pi$. Bottom: IBS synchrotron light curves for the sample inclinations $i=90^{\circ}, 70^{\circ}, 50^{\circ}$; two orbital periods are shown for clarity.

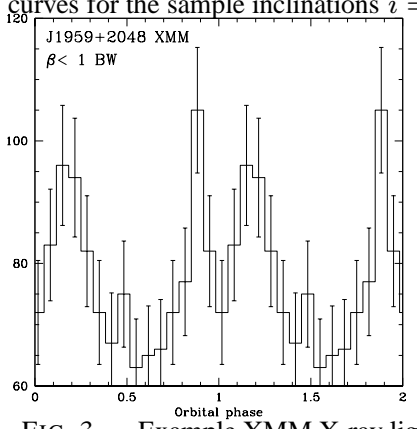

FIG. 3.- Example XMM X-ray light curves, with two periods and phasing as in the lower row of Figure 2. J1959+2048 is a powerful black widow with weak companion wind (low $\beta$ ). PSR J2129-0429 is a low power redback undergoing qRLOF (likely high $\beta$ ). These can be compared with the first and last panels of the bottom row of figure 2 .

where we have assumed an effective albedo $=0$. For a spindown power $\dot{E}$ we may alternatively write a heating efficiency $\eta=L_{H} / \dot{E}$. Physically, we expect this to be modest and several BW/RB do indeed show $\eta \leq 0.1$, however other systems show apparent $\eta \geq 1$ (Breton et al. 2013; Romani. Filippenko \& Cenko 2015); we can consider $\eta$ an alternative heating parameter. To match the observed fluxes the solution also depends on the Roche lobe filling factor $f_{1}$, the system distance, and the interstellar extinction $A_{V}$. In practice, these last three parameters are substantially covariant, while having only a weak affect on determinations of $T_{c}, i$ and $q$.

One challenge to any light curve fitting is the small, but obvious offset between the timing ephemeris and the time of optical maximum. SH14 quote a heating center phase shift
$\Delta \phi=-0.0140 \pm 0.0005$ (ELC fit). In Romani et al. (2015) we found $\Delta \phi=-0.0089$, with very large statistical significance. Any model that does not have such a shift is completely unacceptable. This offset, and similar shifts and asymmetries noted for other BW/RB are prime motivations for an indirect heating model. In our model the heating asymmetry is introduced via $v_{\text {Rel }}$. Note that this is a physical parameter with a meaningful value, and introduces asymmetry without an arbitrary phase shift. Since this parameter dominates the INS fit to the optical light curves, it replaces $\Delta \phi$, leaving the same number of degrees of freedom. More detailed fits (or fits including X-ray light curves, see below) can include $\beta$ or even $\lambda$, but the optical dependence is generally weak.

We thus compare fits with ICARUS-IBS using, as in Romani et al. (2015), Harris BVR color tables from the PHOENIX models tabulated at the Spanish Virtual Observatory (svo2.cab.inta-csis.es). We discuss the color sensitivities and then turn to the fits' dependence on other parameters. Our results are summarized in Table 1 and Figure 5.

\section{1. $T_{D}$, Extinction and Color Terms}

The color as a function of orbital phase should be a powerful constraint on the heating distribution. Several factors typically complicate its use. First, there is inevitably some uncertainty in the observations' zero point calibrations. For example, for the J2215 BVR set, absolute photometry errors may be as large as $0.1 \mathrm{mag}$ (bootstrap estimate, J. Tan, private communication), although night-to-night stability suggests that the relative photometry is considerably better. Also, there is appreciable degeneracy between $T_{e f f}$ and $A_{V}$ (and 

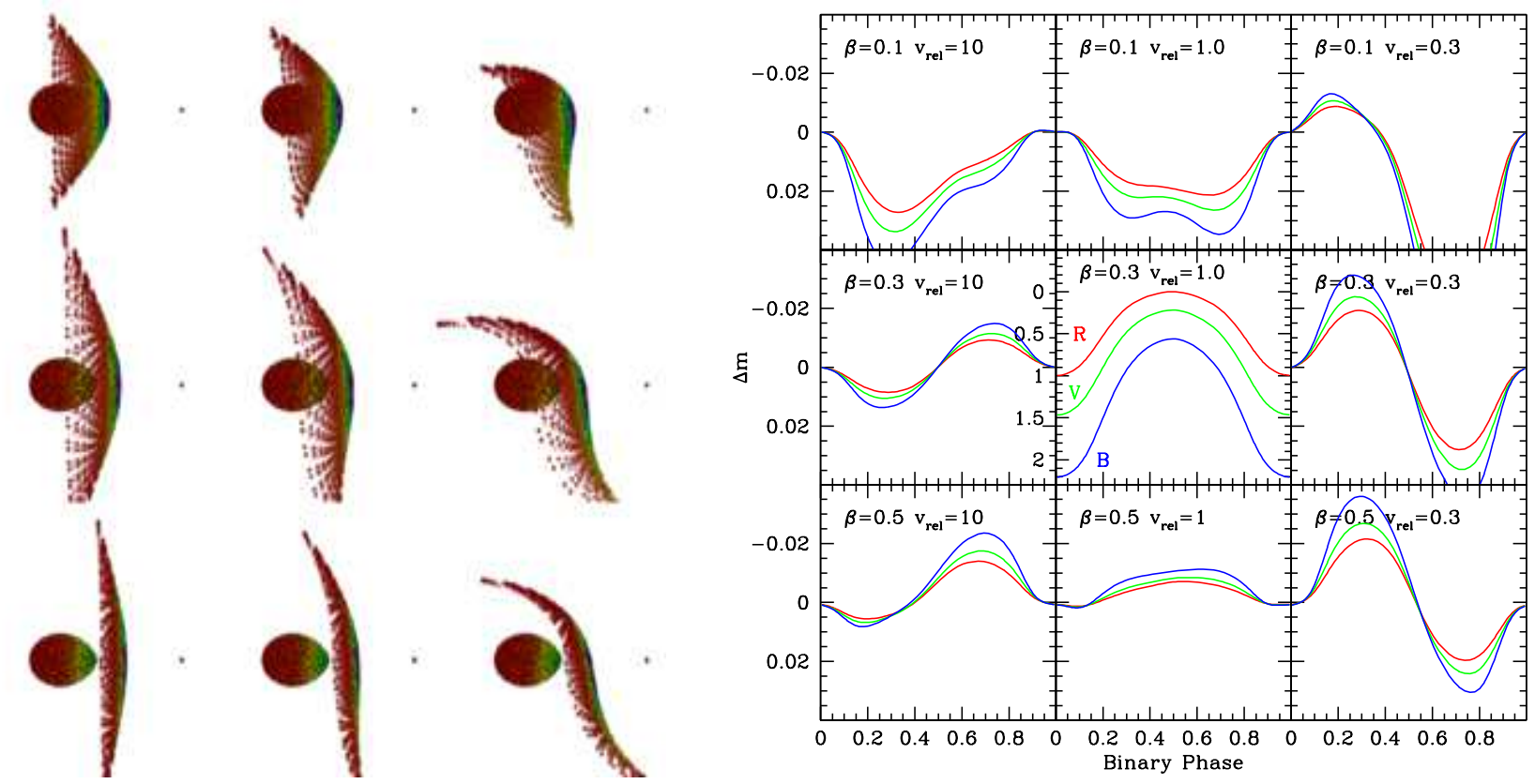

FIG. 4.- Intra-Binary shock geometry dependence on the principal wind parameters. Left: companion wind momentum increases from top to bottom and velocity decreases from left to right. The intrabinary shock is color coded by the fraction of the shocked power hitting the companion surface and the companion is color coded by the local heating power. Right: corresponding BVR light curves for $i=70^{\circ}$. The center panel shows the curves for a $\beta=0.3, v_{\mathrm{Rel}}=1.0$, normalized to the $R$ maximum. The surrounding panels show light curve changes from this case.

the distance modulus DM) - in fact for the $B-V, V-R$ colors in our $T_{\text {eff }}$ range, the degeneracy is particularly bad. If the heating model (or the data calibration) are imperfect, such degeneracy can allow subtle light curve shape disagreements to pull the fit values to incorrect temperatures.

Accordingly it may be useful to use external (nonphotometric) constraints to control some model parameters. For example RGFK15 find effective temperatures $T_{D} \approx$ $9000 \mathrm{~K}$ and $T_{N} \approx 6000 \mathrm{~K}$ from the spectroscopy. This implies a nominal heating efficiency $\eta_{H}=L_{H} / \dot{E} \approx$ $0.48 / I_{45}$ for a direct radiative heating picture. Constraints on the extinction are less direct. The pulsar dispersion measure $\mathrm{DM}=69.2 \mathrm{~cm}^{-3} \mathrm{pc}$, which corresponds to a distance $d=3.0 \pm 0.35 \mathrm{kpc}$ in the NE2001 DM model, converts to $\mathrm{N}_{\mathrm{H}}=2.07_{-0.6}^{+0.9} \times 10^{21} \mathrm{~cm}^{-2}$ (He, Ng \& Kaspi 2013) which corresponds to $A_{\mathrm{V}}=0.72$ (Foight et al 2015). However, more direct estimates from Pan-STARRS photometry (Green et al. 2015) give $A_{V}=0.47$ at $3 \mathrm{kpc}$ and a maximum Galactic $A_{V}=0.71$ in this direction. Unfortunately the existing $\mathrm{X}$-ray exposure is too short to give a constraining absorption measurement. Thus we conclude that $\mathrm{A}_{\mathrm{V}} \leq 0.7$.

In practice we find that unconstrained fits to the SH14 photometry give $T_{D} \approx 13,000 \mathrm{~K}$ and $A_{V} \approx 1.4$ without IBS heating. This indicates that the measured colors are not consistent with the standard atmosphere models, or that the light curve shapes drive the model to artificially high $T_{D}$. We did attempt to see what zero point shifts could drive the best-fit $T_{D}$ down to $9000 \mathrm{~K}$, but the large $\sim 0.1 \mathrm{mag}$ required values resulted in rather poor fits to the light curve shapes.

\subsection{Fitting Results}

In all cases, we find that the fits improve if we add a small positive offset to the measured B magnitudes; in one case a $0.01 \mathrm{mag}$ relative shift in $\mathrm{R}$ is also indicated. Fitting with a symmetric direct heating model is completely unacceptable, with a minimum $\chi^{2}=2400$. Thus at a minimum, we must introduce the (here arbitrary) phase shift of the heating cen- ter by $\Delta \phi=-0.01$. This gives a much more acceptable $\chi^{2}=876$ (Table 1$)$. However, including the IBS reprocessing of the heating (with $\beta=0.5$ ) further improves the fit to $\chi^{2}=729$; the model parameters are listed in Table 1 and the model and fit residuals are shown in Figure 4. This best fit is however, not statistically acceptable since with $271-7=264$ degrees of freedom, this is $\chi^{2} / D o F=2.76$. Certainly a substantial portion of this large $\chi^{2}$ is caused by individual outlier points. Also, the scatter in Figure 5 is larger than expected from the error flags, especially near minimum, which indicates that either the photometric errors of SH14 are underestimated or that there is true stochastic photometric variability. However, inspection of the residuals, especially for $\mathrm{V}$ and $\mathrm{B}$, also shows systematic trends. Clearly our IBS heating model does not give a perfect representation of the true surface temperature distribution. For example the positive $\mathrm{V}$ and negative $B$ residuals around maximum suggest that the nose heating is overestimated by the IBS pattern. Accordingly, the quoted fit statistical errors, even inflated by $\chi^{2} / D_{o F}$, are not a complete description of the uncertainties, and some amendment to the model is needed.

As noted in $\S 4.1$, the incompleteness of the model is also seen from the preference in both the best direct and best IBS models for large $T_{\text {eff }} \approx 12,000-13,500 \mathrm{~K}$ and $A_{V} \approx 1.3$. One worries that the unmodeled effects pulling the $T_{D}$ to such large values may also bias other parameters. Accordingly we also fit while constraining $T_{D}=9000 \mathrm{~K}$. The $\chi^{2}$ values increase, of course, but the best fit baseline temperature $T_{N}$, heating luminosity and $A_{V}$ drop to more reasonable values. The primary geometrical difference is somewhat larger Roche lobe fill factor $f_{1}$.

Note that the IBS fits have significantly lower $\chi^{2}$ than the direct heating models, both with and without $T_{D}$ constraint. These are large $\Delta \chi^{2}>150$ changes relative to the $\Delta \chi^{2} \approx 6-10$ associated with the parameter error ranges. These $1 \sigma$ ranges are projected, multi-parameter errors in all cases. In these fits we have held fixed $\beta=0.5$ and $\lambda=0.7$, since 
TABLE 1

ICARUS MODEL FITS

\begin{tabular}{|c|c|c|c|c|}
\hline Param. & Direct $^{\mathrm{a}}$ & Direct- $T_{D}^{\mathrm{a}}$ & IBS & IBS- $T_{D}$ \\
\hline$i$ & $79.6 \pm 3.2$ & $78.3 \pm 3.1$ & $89 \pm 8$ & $83 \pm 6$ \\
\hline$f_{1}$ & $0.867 \pm 0.004$ & $0.912 \pm 0.004$ & $0.852 \pm 0.006$ & $0.905 \pm 0.004$ \\
\hline$T_{N}(\mathrm{~K})$ & $7670 \pm 76$ & $6421 \pm 23$ & $7290 \pm 79$ & $6416 \pm 58$ \\
\hline$L_{H}\left(10^{34} \mathrm{erg} / \mathrm{s}\right)$ & $29.0 \pm 3.5$ & $5.7 \pm 0.2$ & $32.9 \pm 3.0$ & $11.4 \pm 0.8$ \\
\hline$T_{D}(\mathrm{~K})$ & $13,350^{\mathrm{b}}$ & $9000^{c}$ & $11,710^{\mathrm{b}}$ & $9000^{c}$ \\
\hline$A_{V}$ & $1.39 \pm 0.03$ & $0.75 \pm 0.02$ & $1.25 \pm 0.03$ & $0.79 \pm 0.04$ \\
\hline DM (mag) & $13.76 \pm 0.03$ & $13.56 \pm 0.01$ & $13.62 \pm 0.02$ & $13.51 \pm 0.03$ \\
\hline $\mathrm{dB} / \mathrm{dR}$ & $0.03 / 0.00$ & $0.06 / 0.01$ & $0.04 / 0.00$ & $0.06 / 0.00$ \\
\hline$v_{\text {Rel }}$ & - & - & $0.277 \pm 0.007$ & $0.283 \pm 0.007$ \\
\hline$\chi^{2}$ & 876 & 1228 & 729 & 1018 \\
\hline
\end{tabular}

a Best (arbitrary) phase shift $\delta \phi_{B}=-0.01$ applied.

b Flux weighted Effective $T_{D}$ at $\phi_{B}=0$ computed from $L_{H}$ etc.

c Flux weighted Effective $T_{D}$ at $\phi_{B}=0$ fixed.

the optical light curves depend only weakly on these quantities, and have varied only the $v_{\text {Rel }}$ IBS parameter. Since this parameter replaces the arbitrary phase shift $\Delta \phi$ required by the direct heating models, the fits all have effectively the same number of degrees of freedom. If we do free $\beta$ in the IBS fits, we find $\beta=0.75 \pm 0.3$ and $\beta=0.49 \pm 0.5$ for the IBS and IBS $-T_{D}$ cases, respectively. As expected for such poorly determined parameters, the $\chi^{2}$ decrease is small.

Realistically, the best constraints on the IBS parameters will, in many cases, come from X-ray orbital light curves. In the case of J2215, we have only a low statistics $17 \mathrm{ks}$ ACIS exposure to compare with. Figure 6 shows the X-ray count rate, phased with pulsar superior conjunction at $\phi=0$ along with a curve computed for the IBS tangential emission for the parameters fit to the optical light curves. Although these Xray data are not used in the fit it is encouraging that for these parameters the model predicts a single strong X-ray peak at $\phi \approx 0.25$, in excellent agreement with the data. A high quality X-ray light curve would be very useful to directly constrain the IBS geometrical parameters, as well as measure the radiation spectrum.

\section{CONCLUSIONS}

We have investigated a model in which pulsar radiation is reprocessed through an intrabinary shock before heating a low mass companion. Our shock geometry is controlled by two dimensionless parameters, $\beta$ and $v_{\text {Rel }}$, and is idealized as following the contact discontinuity in the thin-shock limit. When we compute the expected synchrotron emission from the shocked pulsar wind accelerated tangential to the contact discontinuity, we find a variety of asymmetric X-ray light curves, dependent on these two parameters. The companion heating, modeled as a result of direct companion illumination from the prompt post-shock emission also produces a range of asymmetric light curves.

In applying these models to PSR J2215+5135, which has well measured optical light curves and spectra, we find that the IBS model fits the data appreciably better than the direct illumination model, even when the latter is allowed an arbitrary phase shift. This improvement persists even we constrain the fits to match the spectrally determined $T_{D}$ and the expected $A_{V}$ in this direction. Thus the model presents a substantially improved representation of the data - and provides a physically meaningful origin of the observed phase shift of the optical maximum. In addition, the observed X-ray light curve, while of too low statistics to allow a detailed parameter fit, does provide a good match to the IBS light curve expected from the best-fit to the optical data. This is all very encouraging and suggests that $v_{\text {Rel }}$ is a relatively low 0.28 . $\beta$ is larger at $\approx 0.5$ but is not well determined. These nominal values imply a velocity (relative to the companion center, at Roche lobe exit) of $115 \mathrm{~km} / \mathrm{s}$ and a companion mass loss rate of

$$
\begin{gathered}
\dot{M} \approx \beta \dot{E} /\left(c v_{\text {orb }} v_{\text {Rel }}\right)= \\
\left(\beta \dot{E} P_{B} \sin i\right) /\left(2 \pi c^{2} x v_{\text {Rel }}\right)=1.7 \times 10^{-9} I_{45} M_{\odot \mathrm{y}^{-1}}
\end{gathered}
$$

Thus, the shape and sweepback of this IntraBinary Shock implies that, at the present companion mass-loss rate, evaporation has a characteristic timescale $\tau_{\text {evap }}=M_{c} / \dot{M} \approx 150 \mathrm{Myr}$. If this rate persists, we expect $\mathrm{J} 2215$ to be an isolated MSP in $<$ Gy. It should be noted, however, that J2215 has parameters rather similar to those of the 'transitioning' MSP J1023+0038 and so might spend time in an accretion phase, suppressing pulsar irradiation and leading to a lower mass loss rate. The relatively large $\beta$ preferred by our model fits, suggest that the pulsar wind could indeed be overwhelmed by a fluctuation in mass-loss rate, burying the pulsar.

Despite these successes, the model is clearly not complete, as shown by the large residual $\chi^{2}$. Some of this is due to understated errors, and individual outlier points (possibly indicating flare events as seen, e.g. for PSR J1311-3430, Romani et al 2105). However, systematic light curve shape residuals, especially for models constrained to match the spectral temperature, indicate deficiencies in the computed heating pattern. Also, although zero-point errors in the photometry undoubtedly play a role, the preference of the fits for high $T_{D}$ indicates an incorrect heating distribution. Finally, the IBS, while capturing a much larger fraction of the pulsar spin-down power, does not focus this power to the companion surface. Indeed, our assumed prompt radiation, computed with the forward shock jump conditions, takes the incident pulsar power and deflects it away from the shock normal (and thus, for most positions on the IBS, further from the companion). Thus we find that re-processing the pulsar power through the IBS requires up to $2 \times$ larger pulsar luminosity (for this prompt post-shock illumination picture) than direct heating. For the best-fit IBS model we infer an efficiency $\eta=4.4 / I_{45}$ and for the $T_{D}$-constrained IBS model $\eta=1.5 / I_{45}$. These factors are for a $\sin ^{2}$ wind flow and are only modestly reduced for a $\sin ^{4}$ distribution. Finally, while we have matched the apparent asymmetry for $\mathbf{J} 2215$, some other wind-driving pulsars display much larger optical heating asymmetries, that would 

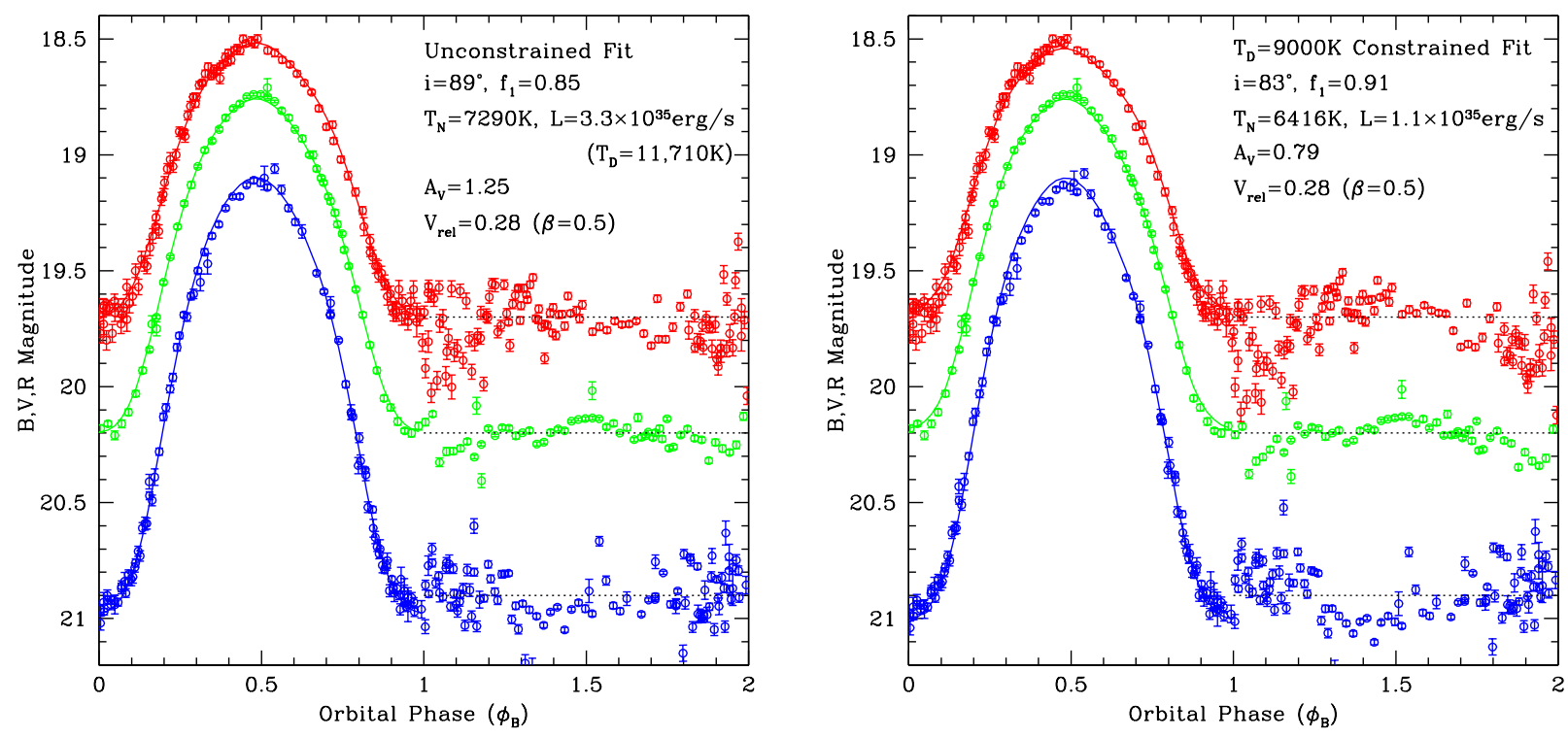

FIG. 5.- SH14 BVR light curves with IBS-fit model (1st period). The second period shows the fit residuals (expand $3 \times$ ). Left: best fit model, Right: best fit $T_{D}$ constrained model.

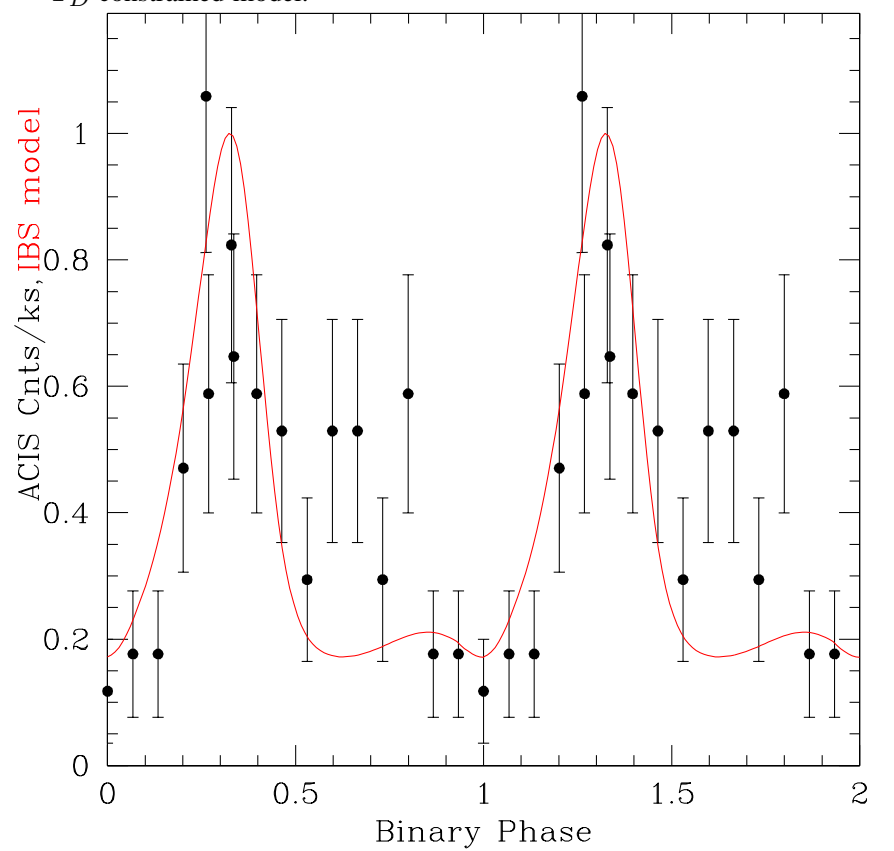

FIG. 6.- J2215+5135 ACIS X-ray light curve (points) with the predicted IBS tangential synchrotron light curve for the parameters of the model which best fit the optical data.

be difficult to produce in this model even with small $v_{\text {Rel }}$.

We conclude that while IBS-reprocessing through a swept back model can be a viable solution for some wind-driving pulsars, an additional physical ingredient is likely needed to fully match the heating data and to explain particularly extreme cases.

The most likely culprit is ducting by companion magnetic fields. There is in fact good reason to believe that substantial fields can be supported by the companion. BW and RB are short period, tidally locked binaries so the secondaries are, by definition, rapidly rotating stars. Also, since night side temperatures of black widows and redbacks are appreciably higher than expected for the unperturbed star, rapid motion must be advecting heat to the night sides. These convective motions in the presence of rapid spin give a plausible dynamo origin for large, dynamic B fields.

With a typical standoff distance $r_{0} \sim 0.3 R_{\odot} \sim 2 \times$ $10^{10} \mathrm{~cm}<a$, companion-supported fields with a dipole of strength $B_{C}$ and coherence scale $r_{C}$ can channel the wind flow if

$$
\left[B_{C}\left(r_{0} / r_{C}\right)^{3}\right]^{2} / 8 \pi>\dot{E} /\left(4 \pi a^{2} c\right)
$$

or $B_{C}>8\left(r_{0} / r_{C}\right)^{3} \mathrm{G}$. So the dominance depends on the large scale coherence of the companion dipole field located near $r_{C}(0)$. For J1311-3430 Romani, Filippenko \& Cenko (2015) observed apparently magnetically-driven flares with the surface heating and flux giving a characteristic size $\sim$ $r_{C}(0) / 3 \sim 0.02 a \sim 0.1 r_{0}$ and energy density equivalent to $B_{C} \sim 10 \mathrm{kG}$, which could be dynamically significant at the IBS standoff distance. If similar field exist in the companions of other BW/RB we may expect them to redirect the energy released in an IBS. We can then imagine IBS particles precipitating from a cross sectional area $\sim \pi r_{0}^{2}$ to the companion surface, inducing heating at the field line foot points. Such local heating would inevitably induce temperatures higher than the mean $T_{D}$ and will, in general, have foot point hot-spots offset from the sub-pulsar point. Indeed if the local field is not largely dipolar we may have precipitation at many poles and a complex, offset heating pattern. It remains to be seen if a detailed model of such field-mediated heating, which could be fit to observed light curves and spectra, could have useful predictive power.

We thank Hongjun An and Yajie Yuan for helpful discussions about shock interactions, Josh Tan for insight into the SH14 data and Rene Breton for advice on the ICARUS code.

\section{REFERENCES}

Aldcroft, T., Romani, R. V. \& Cordes, M. 1992, ApJ, 400, 638 Bellm, E. C., Kaplan, D. L., Breton, R. P. et al. 2016, ApJ, 816, 74 Bosch-Ramon, V. Barkov., M. V. \& Perucho M. 2015, AA, 577, 89 Breton, R. P., et al. 2013, ApJ, 769, 108
Bogdanov, S., Archibald, A. M., Hessels, J. W. T., et al. 2011, ApJ, 742, 97 Bogovalov, S. V., Khangulya, K., Koldoba, A. V., Ustyugova, G. V. \& Aharonian, F. A. 2012, MNRAS, 419, 4326

Callanan, P. J., van Paradijs, J. \& Rengelink, R. 1995, ApJ 439, 928 
Canto', J., Raga, A. C., \& Wilkin, F. P. 1996, ApJ, 469, 729.

Djorgokski, S., \& Evans, C.R. 1988, ApJ 335, L61

Foight, D. R., Guever, T., Oezel, F. \& Slane, P. O. 2015, ArXiv150407274

Gentile, P. A., Roberts, M. S. E., McLaughlin, M. A., et al. 2014, ApJ, 783, 69

Green, G. M., Schlafly, E. F., Finkbeiner, D. P. et al. 2015, ApJ, 810, 25

He, C., Ng, C.-Y., \& Kaspi, V. M. 2013, ApJ, 768, 64.

Huang, R. H. H., Kong, A. H. K., Takata, J., et al. 2012, ApJ, 760, 92

Husser, T.-O., Wende-von Berg, S., Dreizler, S., et al. 2013, AA, 533, A6

Komissarov, S S., \& Lyutikov, M. 2011, MNRAS, 414, 2017

Orosz, J. A., \& Hauschildt, P. H. 2000, AA, 364, 265

Parkin, E. R., \& Pittard, J. M. 2008, MNRAS, 388, 1047

Roberts, M. S. E., McLaughlin, M. A., Gentile, P. A. et al. 2014, AN, 335, 315
Roberts, M. S. E., McLaughlin, M. A., Gentile, P. A. et al. 2015, ArXiV, 1502.07208

Romani, R. W. 2015, ApJ, 812, L24

Romani, R. W., Filippenko, A. V., \& Cenko, S. B. 2015, ApJ, 804, 115.

Romani, R. W., Graham, M. L., Filippenko, A. V., \& Kerr, M. 2015, ApJ, 809,10

Schroeder, J., \& Halpern, J. P. 2014, ApJ, 793, 78

Stappers, B.W, van Kerkwijk, M. H., Bell, J.F. \& Kulkarni, S. R. 2001, ApJ, 548,183

Tchekhovskoy, A., Spitkovsky, A. \& Li, J. G. 2013, MNRAS, 435, L1 van Kerkwijk, M. H., Breton, R. P., \& Kulkarni, S. R. 2011, ApJ, 728, 